\title{
The Reverse Operation Of Knot Digraph Notation
}

\author{
Tamer UĞUR ${ }^{1}$ Ceren Sultan ELMALI ${ }^{2 *}$, Ferit YALAZ ${ }^{3}$ \\ ${ }^{2}$ Department of Mathematics, Faculty of Science and Arts, Atatürk University, 25240 Erzurum TURKEY \\ ${ }^{2}$ Department of Mathematics, Faculty of Science and Arts, Erzurum Technical University, 25100 Erzurum-TURKEY \\ ${ }^{3}$ Graduate School of Natural and Applied Science, Erzurum Technical University, 25100 Erzurum-TURKEY
}

\begin{abstract}
It is well known that bitopologies associated with knot digraphs is finded by using knot digraph notation. In this work, we have developed a method that we called reverse of knot digraph notation to find out which knot belongs to when a bitopology associated with the knot is given.
\end{abstract}

\section{Introduction}

The notion of bitopological space $\left(X, \tau_{1}, \tau_{2}\right)$, that is, a set $X$ equipped with two arbitrary topologies $\tau_{1}, \tau_{2}$ was first formulated by J.C.Kelley [4] Girija and Plakkat showed that bitopology is get from a digraph which has orientation by using quasi-pseudo metric entitled with 'Bitopological space associated with digraphs' [3] in 2013. A quasi pseudo metric on nonempty set $X$ is a nonnegative real valued function $\rho$ on $X \times X$ such that $\rho(x, x)=0, \forall x \in X$ and $\rho(x, z) \leq \rho(x, y)+\rho(y, z), \forall x, y, z \in X$ associated with $\rho$ there is another quasi pseudometric $q$ such that $\rho(x, y)=$ $q(y, x)$.In this study the authors is called digraph if the vertex of graph is reachable from edge of it. Let be $V$ the vertex set of a digraph. The function $p: V \times V \rightarrow \mathbb{R}$ and $q: V \times V \rightarrow \mathbb{R}$ defined by

$$
p(x, y)=\left\{\begin{array}{rr}
0, & \text { If } x \text { is reachable from } y \\
1, & \text { otherwise }
\end{array}, q(x, y)=\left\{\begin{array}{lr}
0, & \text { If y is reachable from } x \\
1, & \text { otherwise }
\end{array}\right.\right.
$$

respectively. These functions are quasi-pseudo metrics on $V$ and induces a unique bitopology on $V$ with $S_{p}(x, \varepsilon)=\{y: p(x, y)<\varepsilon\}$ and $S_{q}(x, \varepsilon)=\{y: q(x, y)<\varepsilon\}$ as a basis. The collection of all open $\rho$-balls forms a basis for a topology on $X$.Let this topology be denoted by $\tau_{1}$. Similarly the collection of all open $q$-balls forms a basis for a topology on $X$. Let this topology be denoted by $\tau_{2}$. Thus we get the bitopology $\left(X, \tau_{1}, \tau_{2}\right)$. A bitopological space $\left(X, \tau_{1}, \tau_{2}\right)$ is a set $X$ with two topologies, $\tau_{1}$ and $\tau_{2}$, on it.

We know that this method can be applied for the oriented knot graph which is obtained form regular diagram of a knot. This process is called knot digraph notation. We know that a bitopology matched with any knot by using knot digraph notation. In this work, we have developed an algorithm. The knots that correspond to bitopological spaces will be found with the aid of this algorithm. We called this algorithm as 'reverse operation of knot digraph notation'.

\section{Knot and Knot Graphs}

A knot is a simple closed curve obtained by embedding of the circle $S^{1}$ into $R^{3}$ (or $\mathrm{S}^{3}$ ) [1]. A regular projection of knots or links on a 2-dimensional plane divides the plane into several regions, each of which is homeomorphic to an open disk. It is a frequently used method to separate these regions (domains) into two classes, white-black or shadedunshaded or shadow-unshadow regions, in the study of the knot theory.

A regular projections of knot $K$ divides plane into $n+2$ regions where $\mathrm{n}$ is the number of double point $A\left(A_{1}, A_{2}\right.$, $\left.\ldots, A_{n}\right)$. The Figure 1 show that the number of double point of Trefoil knot is 3 . Therefore The number of regions is 5 .

\footnotetext{
${ }^{*}$ Corresponding Author: ceren.elmali@erzurum.edu.tr
} 




Figure 1. The number of double point of Trefoil knot

Starting with the outer most region, we can color the regions either black or white where neighboring regions are never this same color. We are labeled white and black regions, respectively, such that $\alpha=\left\{\alpha_{1}, \alpha_{2}, \ldots, \alpha_{k}\right\}$ and $\beta=$ $\left\{\beta_{1}, \beta_{2}, \ldots, \beta_{l}\right\}$. The point at infinity is contained in a region of black. Take points $P_{i} \in \alpha_{i}(i=1,2, \ldots, k)$ and connect this points by $n$ - non-intersection $\operatorname{arcs} d_{1}, d_{2}, \ldots, d_{n}$ in such a way each $d_{t}$ corresponds to $A_{t}(t=1,2, \ldots, n)$ and $P_{i}$ and $P_{j}$ connected by $d_{t}$, if and only if $\alpha_{i}, \alpha_{j}$ have a common double point $A_{t}$ on their boundaries. In this way, we obtain from regular projection of the knot a plane graph $G$. From same consideration about class $\beta$, we get another graph $G^{d}$. We call this the dual graph of $G$. This method is called as Tait's method. Figure 2(a) and 2(b) show that graph and dual graph of Trefoil knot, respectively,



(a)

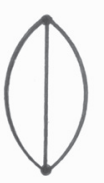

(b)

Figure 2. Graph and dual graph of Trefoil knot

The regions of class $\alpha$ in all can be considered as a projection of a surface spanning $K$ which is twisted $180^{\circ}$ at each double point of projection. From this consideration, we define the sign of $d_{t}$ in such a way that it is + or - according as the surface is twisted at $A_{t}$ as the right screw or the left one, respectively (Figure 3 ).

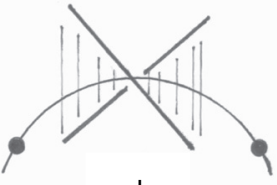

$+$

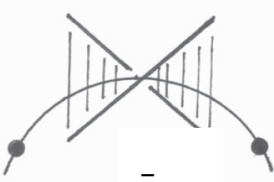

Figure 3. + or - according as the surface is twisted at $A_{t}$

It is well known that every deformation of a knot is equivalent to a combination of the fundamental deformation (Reidemeister Movies) $\Omega_{1}, \Omega_{2}$ and $\Omega_{3}$ in [7].
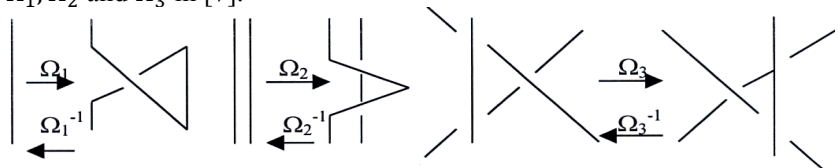

Figure 4. Reidemeister Movies

These fundamental deformations can be translated into the operations on graphs as follows [8].





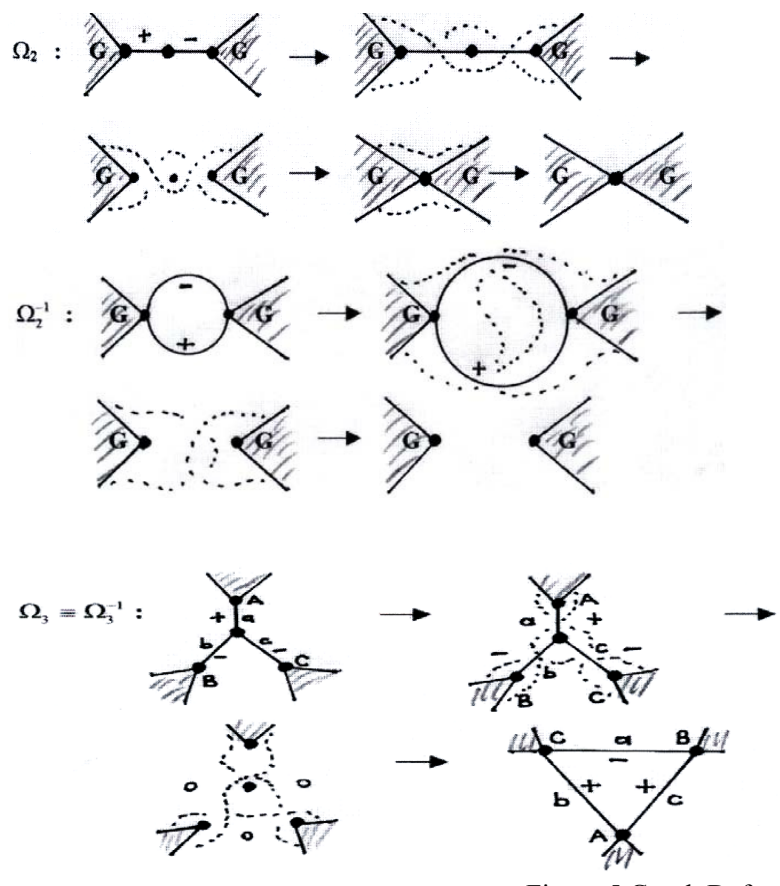

Figure 5.Graph Deformations

We recommend $[2,5,6]$ for details about this section.

\section{Knot digraph Notation}

Firstly, signed graph is obtained from regular diagram of a knot by using Tait methods explained in Section 2.Secondly, orientation is given depending on the signed of the signed knot graph as Figure 6.If the signed of edge is + , orientation of this edge is given from a to $b$. If the signed of edge is -, orientation of this edge is given from $b$ to $a$. So we can get two directed graphs, when we consider graph and dual graph of a knot. We called this directed graph as knotdigraph.

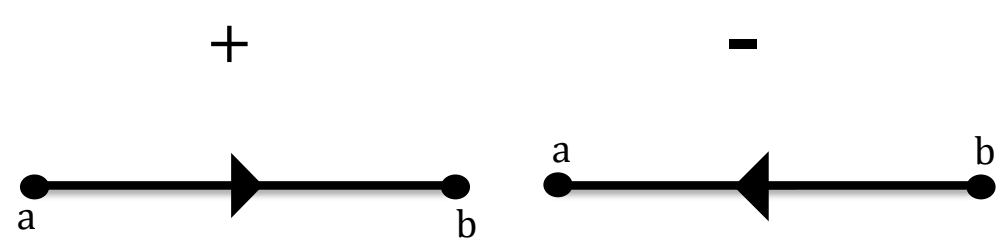

Figure 6. Orientation of knot graph depend on the signed of the signed knot graph

After that, every vertices of knot digraph are labelled by lowercases. Finally, the base is obtained from knot digraph by using quasi-pseudo metric for bitopology. Thus, every knot is matched with bitopology obtained by using this method.We called this method as Knot digraph Notation

\section{The Knots Obtaining with the Help of Bitopology}

Definition 4.1: Let $\left(X, \tau_{1}, \tau_{2}\right)$ is a bitopological space. The set $\tau_{1}$ has the largest number of elements, except the $\mathrm{X}$ set, is called the strong set of $\tau_{1}$ and is denoted by $\overline{\tau_{1}}$. Similiarly, the set which is the largest number of elements, except $X$ is called the strong set of $\tau_{2}$ and is denoted by $\overline{\tau_{2}}$.

Definition 4.2: Let $\left(X, \tau_{1}, \tau_{2}\right)$ is a bitopological space. The set of $\tau_{1}$ with at least one element excluding the null set is called the weak set of $\tau_{1}$ and is denoted by $\tau_{1}$. Similiarly, the set is called the weak set of $\tau_{2}$ with at least one element excluding the null set, denoted by $\underline{\tau_{2}}$. 
The number of elements of $\overline{\tau_{1}}$, the strong set of $\tau_{1}$ topology, is the corner number (node) of the knot digraph corresponding to the bitopological space of $\left(X, \tau_{1}, \tau_{2}\right)$. The number of elements of $\overline{\tau_{1}}$ is denoted by $s\left(\overline{\tau_{1}}\right)=V$. The number of elements of the set $\underline{\tau_{2}}$, the weak set of $\tau_{2}$ topology, shows how many impairment are made between digits in the knot digraph corresponding to the bitopology $\left(X, \tau_{1}, \tau_{2}\right)$. We denote the number of elements of $\underline{\tau_{2}}$ by $s\left(\underline{\tau_{2}}\right)=B$

The regular diagrams of $m_{n}$ knots corresponding to the bitopologies using loop distortion with $m=3,4 \ldots 10$ and $n=1,2,3$ will be found by the following six steps.

Step 1.A node corresponding to the bitopological space $\left(X, \tau_{1}, \tau_{2}\right)$ is marked by $s\left(\overline{\tau_{1}}\right)=V$. All points that are made are numbered $1,2, \ldots, \mathrm{V}$ as shown in Figure 7 in clockwise direction.

Figure 7. Corner number of the knot given the bitopology

Step 2. The number of element $\varepsilon_{k}^{i j}$ of $\underline{\tau_{2}}$ shows how many corruptions are made between $\mathrm{i}$ and $\mathrm{j}$. For example, while the $\varepsilon_{1}^{13}$ element shows an impairment made between 1 and 3 corners, the $\varepsilon_{2}^{13}$ element shows two impairments made between 1 and 3 corners. This impairment is as following Figure 8.

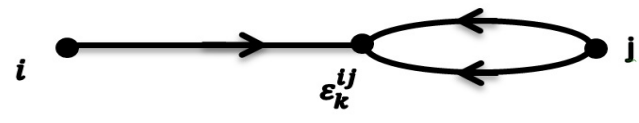

Figure 8. Cycle degradation

Step 3. $V+2 B-1$ formula gives the number of edges of knot digarph. Namely it gives crossing number of given knot Step 4. After process of the impairment $V-1$ edges are added clockwise from the numbered edge by 1 .

Step 5. Graph movements transform digraph into each other. Especially, the movement shown in the following Figure 9 is often used.
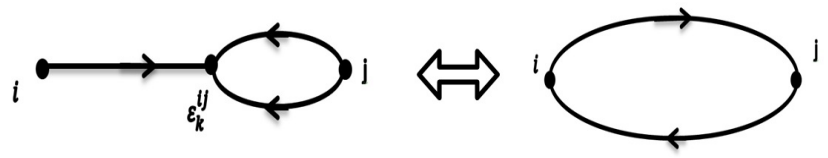

Figure 9. Cycle disturbance and cycle

Step 6. In the first five steps a knot digraph corresponding to the given bitopology is found. We can get knot form this knot digraph by using Tait's method.

Now, the knots that correspond to the following bitopological spaces will be found with the aid of the given algorithm.

Example: Let $\left(X, \tau_{1}, \tau_{2}\right)$ is a bitopological space such that

which knot corresponds to this bitopology?

$$
\begin{gathered}
\tau_{1}=\{X, \emptyset,\{1\},\{1,2\},\{1,2,3\}\} \\
\tau_{2}=\left\{X, \emptyset,\left\{2,3, \varepsilon_{1}^{13}\right\},\left\{3, \varepsilon_{1}^{13}\right\},\left\{\varepsilon_{1}^{13}\right\}\right\}
\end{gathered}
$$

1. $\overline{\tau_{1}}=\{1,2,3\}, s\left(\overline{\tau_{1}}\right)=V=3$. So let's place 3 points of flattening and give this number in clockwise direction as in Figure 10.

\section{$\bullet^{2}$}

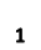

Figure 10.Placement of the points

2. $\underline{\tau_{2}}=\left\{\varepsilon_{1}^{13}\right\}, s\left(\underline{\tau_{2}}\right)=B=1$. Then an impairment is placed between points 1 and 3 as in Figure 11 .

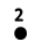

1

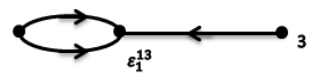

Figure 11. Placement of the impairment

3. $V+2 B-1=3+2-1=4$, this shown that drawn digraph has got 4 edges 
4. $V-1=3-1=2$, this shown that 2 edges will be added clockwise from 1 as in Figure12.



Figure 12. Placement of the edges

5. The impairment between points 1 and 3 is transformed by using graph movies as in Figure 13 .

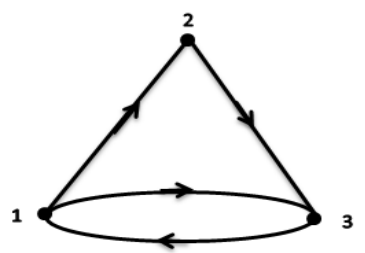

Figure 13. Transformed digraph

6. Using the Tait method, $\mathbf{4}_{\mathbf{1}}$ knot are obtained.

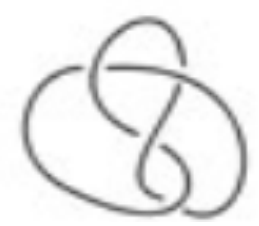

Figure 14. Regular diagram of $\mathbf{4}_{\mathbf{1}}$ knot

\section{References}

1. Alexander, J.W., G.B., On types of knotted curve. Ann. Math., 28, 562-586, 1956.

2. Bankwitz, C., Uber die Torsionszahlen der alternierden Knoten. Math. Ann., 103, 145-161, 1930.

3. Girija B., Pilakkat R., Bitopological spaces associated with digraphs, South Asian Journal of Mathematics, Vol.3 (1):56-65, 2013.

4. Kelley J.C., Bitopological Spaces, Proc. London Math.(3), 13, 71-89, 1963.

5. Murasugi, K., On invariants of graphs with applications to knot theory. Trans. Amer. Math. Soc., 314, 1-49, 1989.

6. Murasugi, K., Knot Theory and Its Application.Boston,1993.

7. K. Reidemeister: Knotentheorie, Julius Springer, 1932.

8. Yajima T., Kinoshita S., On the graphs of knots, Osaka Math. J., 9, 155-163, 1957. 